Pacific Journal of Mathematic 


\title{
CLOSURE PROPERTIES IN RADICAL THEORY
}

\author{
E. P. ARMENDARIZ
}

The purpose of this paper is two-fold. First, we show that certain known properties of associative radicals cannot be extended to the class of all rings. Secondly, we investigate classes of (associative) rings closed under ideals and subdirect sums in relation to radical and semisimple classes. Much of this is motivated by results obtained for torsion theories in categories of modules by S. E. Dickson and J. P. Jans. We remark that Dickson's work was developed in the more general setting of Abelian categories.

A nonnull class $\mathscr{M}$ of (not necessarily associative) rings is a radical class in the sense of Amitsur-Kurosh $[2,12]$ if it has the following properties:

(i) Every homomorphic image of an $\mathscr{C}$-ring is an $\mathscr{H}$-ring.

(ii) Every ring $R$ contains an $\mathscr{C}$-ideal, called the $\mathscr{L}$-radical of $R$ and denoted by $m(R)$, which contains every other $\mathscr{L}$-ideal of $R$.

(iii) The factor ring $R / m(R)$ has no nonzero $\mathscr{C l}$-ideals (i.e., $R / m(R)$ is $\mathscr{L}$-semisimple).

A nonnull class $\mathscr{M}$ may satisfy only condition (i), in which case $\mathscr{A}$ can be embedded in a minimal containing radical class by a method due to Kurosh [12], and refined by Anderson, Divinsky, and Sulinski [5]. Define $\mathscr{C}_{1}=\mathscr{C}$ and assuming $\mathscr{C}_{\alpha}$ defined for all ordinals $\alpha<\beta$, define $\mathscr{C}_{\beta}=\{K$ : every nonzero homomorphic image of $K$ contains a nonzero ideal belonging to $\mathscr{C}_{i}$ for some $\left.\delta<\beta\right\}$. Then $\mathscr{L} \mathscr{C}=\bigcup_{\alpha} \mathscr{C}_{\alpha}$ is the desired radical class, called the lower radical class determined by $\mathscr{M}$.

Assume the class $\mathscr{M}$ satisfies the condition

(iv) Every nonzero ideal of an $\mathscr{M}$-ring has some nonzero $\mathscr{C}$ ring as a homomorphic image.

In this case the class $\mathscr{U} \mathscr{C}=\{K: K$ has no nonzero homomorphic images in $\mathscr{A}\}$ is a radical class [12], called the upper radical class determined by $\mathscr{C}$. A special instance of (iv) occurs when the class $\mathscr{C}$ is hereditary: every ideal of an $\mathscr{C}$-ring is an $\mathscr{C}$-ring.

2. Two examples. For a radical class $\mathscr{C}$ in the class $\mathscr{A}$ of associative rings, Amitsur [2] has established the equivalence of the two conditions:

$(\alpha) \mathscr{M}$ is hereditary.

$(\beta)$ For every $K \in \mathscr{A}$ and every ideal $I$ of $K, m(I)=I \cap m(K)$. This equivalence was extended to the class $\mathscr{B}$ of alternative rings 
in [4] by showing that if $K \in \mathscr{B}, I$ an ideal of $K$, and $\mathscr{C l}$ a radical class in $\mathscr{B}$, then $m(I)$ is an ideal of $K$. This implies in particular that the class $\mathscr{S} \mathscr{C l}$ of $\mathscr{C}$-semisimple rings is hereditary.

Our first example establishes the nonequivalence of $(\alpha)$ and $(\beta)$ in the class of all rings. This example was used in [6] to show that not every semisimple class is hereditary. Note that $(\alpha)$ always implies $I \cap m(K) \subseteq m(I)$ for $I$ an ideal of $K$.

EXAMPLe 2.1. Let $\mathscr{Z}$ denote the class of zero rings (i.e., $R^{2}=0$ ). Then $\mathscr{Z}$ is hereditary and homomorphically closed. Forming $\mathscr{L} \mathscr{Z}$ in the class of all rings, we can assert by [9, Th.3] that $\mathscr{L} \mathscr{Z}$ is a hereditary radical class. Now take $K$ to be the ring generated over $G F(2)$ by the symbols $u, v, w$ subject to: $u^{2}=0, u v=v u=u, u w=$ $w u=v w=w v=v^{2}=v, w^{2}=w$. Then the only proper ideal $\neq 0$ of $K$ is $I=\{0, u, v, u+v\}$, and $J=\{0, u\}$ is an ideal of $I$ but not of $K$. Now $K$ has no nonzero ideals in $\mathscr{L} \mathscr{Z}$, as $K / I \cong I / J \cong G F(2)$, and certainly $G F(2) \notin \mathscr{L} \mathscr{Z}$ (a simple ring $S$ is in $\mathscr{A} \mathscr{A}$ if and only if $S$ is in $\mathscr{\mathscr { C }}$ ). Thus $z(K)=0$. However $J J=0$ so that $J \in \mathscr{L} \mathscr{Z}$ and hence $z(I)=J \neq z(K) \cap I=0$.

Our next example involves the class $\mathscr{L} \mathscr{Z}$ also. An ideal $I$ of a ring $K$ is prime in case $J, N$ ideals of $K$ and $J N \subseteq I$ implies $J \subseteq I$ or $N \subseteq I$. (Here $J N$ can denote either the set product or the ideal generated in $K$ by the set product). A ring $K$ is prime if 0 is a prime ideal of $K$, while $I$ is a prime ideal of $K$ if and only if $K / I$ is a prime ring. As is known, the lower radical class determined by the zero rings in the class $\mathscr{A}$ of associative rings coincides with the upper radical class determined in $\mathscr{A}$ by the prime rings in $\mathscr{A}$. The example will establish that this is not the case in the class of all rings; however, it shows even more. For [5, Th.2] states that if $\mathscr{C l} \leqq \mathscr{A}$ is hereditary, homomorphically closed and contains $\mathscr{Z}$, then $\mathscr{L} \mathscr{C}=\mathscr{C}_{2}$. This cannot be extended to all rings. Specifically we have the following, where $\mathscr{P}=$ prime rings.

Proposition 2.2. In the class of all rings, $\mathscr{P} \nsubseteq \mathscr{S}(\mathscr{L} \mathscr{Z})$ hence $\mathscr{L} \mathscr{Z} \neq \mathscr{U} \mathscr{P}$. However $\mathscr{U} \mathscr{P}=\mathscr{Z}{ }_{2}$ so that $\mathscr{U} \mathscr{P} \cong \mathscr{L} \mathscr{Z}$ and $\mathscr{L} \mathscr{2} \neq \mathscr{Z}_{2}$.

Proof. Assume for the moment that $\mathscr{T} \nsubseteq \mathscr{S}(\mathscr{L} \mathscr{Z})$. If $\mathscr{L} \mathscr{Z}=$ $\mathscr{U} \mathscr{P}$ then $\mathscr{S}(\mathscr{L} \mathscr{Z})=\mathscr{S}(\mathscr{U} \mathscr{P})$. But then $\mathscr{P} \subseteq \mathscr{S}(\mathscr{U} \mathscr{P})$ leads to a contradiction. Next, if $K \in \mathscr{Z}_{2}$ then, since every nonzero image of $K$ has a nonzero $\mathscr{Z}$-ideal, certainly $K \in \mathscr{C} \mathscr{P}$. We establish the opposite inclusion, using a standard argument, by showing that if $K$ has no nonzero $\mathscr{Z}$-ideals then $K$ has a nonzero image in $\mathscr{P}$. Let $0 \neq a_{\vartheta} \in K$. Then $\left(a_{\vartheta}\right)^{2} \neq 0$, where $\left(a_{\vartheta}\right)$ denotes the ideal generated by 
$a_{0}$ in $K$. Selecting $0 \neq a_{1} \in\left(a_{0}\right)^{2}$, we get $\left(a_{1}\right)^{2} \neq 0$, and continuing we obtain a sequence $\left\{a_{n}\right\}$ of nonzero elements such that $a_{i} \in\left(a_{i-1}\right)^{2} \neq 0$. By Zorn's lemma, select an ideal $I$ of $K$ maximal relative to $I \cap\left\{a_{n}\right\}=\varnothing$. Then it follows that $I$ is a prime ideal of $K$; indeed, if $J / I, N / I$ are nonzero ideals of $K / I$ then $J, N$ both intersect $\left\{a_{n}\right\}$. Thus $a_{i} \in J, a_{j} \in N$ with $i \leqq j$ so that $\left(a_{j}\right) \leqq\left(a_{i}\right)$. Then $\left(a_{j}\right)^{2} \leqq J N$ hence $a_{j+1} \in J N$ and $a_{j+1} \notin I$; i.e., $(J / I)(N / I) \neq 0$.

It remains to show then that $\mathscr{P} \nsubseteq \mathscr{S}(\mathscr{F})$, and to do this we proceed as in Example 2.1, letting $R$ be the ring generated by $x, y, z$ over $G F(2)$ subject to: $x^{2}=0, y^{2}=y x=x y=x, y z=z y=x z=z x=y$, $z^{2}=z$. It is readily verified that the only proper ideal of $R$ is $I=$ $\{0, x, y, x+y\}$ and that $R^{2}, R I=I R, I^{2}$ are all nonzero. Thus $R \in \mathscr{S}$. Now $I$ contains $J=\{0, x\}$ as its only ideal and both $J$ and $I / J$ are zero rings. Thus $I \in \mathscr{Z} \subseteq \mathscr{L} \mathscr{Z}$ so that $R$ is not $\mathscr{L} \mathscr{Z}$-semisimple.

We conclude this section with a final remark. In the class of alternative rings let $\mathscr{C}$ consist of all simple rings with unit. As shown in [4], $\mathscr{C} \mathscr{C}$ is a hereditary radical class. Example 2.1 shows that this is not true in the class of all rings since in that example both $K$ and $I$ have $G F(2)$ as an image, while $J \in \mathscr{Z}$, hence is the radical of $I$ relative to this radical class.

3. Hereditary radical classes. Unless noted otherwise, all rings in this and $\S 4$ will be associative. An ideal $I$ of a ring $K$ is large in case $I \cap A \neq 0$ for each ideal $A \neq 0$ of $K$. For a class of rings $\mathbb{C}$ we consider the property:

( $\lambda$ ) If a ring $K$ contains a large $/ \mathscr{C}$-ideal then $K \in \mathscr{l}$.

THEOREM 3.1. Let $\mathscr{R}$ be a radical class with semisimple class $\mathscr{S}$. Then $\mathscr{R}$ is hereditary if and only if $\mathscr{S}$ has property $(\lambda)$.

Proof. Assume first that $R$ is hereditary and let $K$ be a ring having a large $\mathscr{S}$-ideal $I$. As noted in $\S 2, I \cap r(K)=r(I)=0$ since $I \in \mathscr{S}$, and since $r(K)$ is an ideal of $K$ it follows that $r(K)=0$, so $K \in \mathscr{S}$.

Now suppose $\mathscr{S}$ has $(\lambda)$. If $\mathscr{R}$ is not hereditary there is a ring $K \in \mathscr{R}$ with an ideal $I \notin \mathscr{R}$ so that $r(I) \neq I$. Since $r(I)$ is an ideal of $I$ [4, Th. 1], and $\mathscr{R}$ is homomorphically closed, it suffices to assume that $r(I)=0$, i.e., $I \in \mathscr{S}$. Choose an ideal $M$ of $K$ maximal relative to $M \cap I=0$. Then, considering $I$ an ideal of $K / M \neq 0$, the maximality of $M$ ensures $I$ is large in $K / M$. Then $K / M \in \mathscr{R} \cap \mathscr{S}=\{0\}$, a contradiction. Hence $\mathscr{R}$ is hereditary.

In $\S 2$ we made use of the result of Hoffman and Leavitt which states that for a class /l homomorphically closed and hereditary (though not necessarily consisting of only associative rings), each $\mathbb{K}_{\alpha}$ 
in the lower radical construction is hereditary and hence $\mathscr{L} \mathscr{C}$ is hereditary. We establish this for associative rings using Theorem 3.1. At the same time we must appeal to [4, Th. 1] which states that if $\mathscr{C}$ is homomorphically closed then $\mathscr{L} \mathscr{C}=\left(\bigcup_{i=1}^{\infty} \mathscr{C}_{i}\right)_{2}$ for associative rings. Accordingly, let $\mathscr{C}=\mathscr{C}_{1}$ be homomorphically closed and hereditary and let $\mathscr{T}=\mathscr{S}(\mathscr{L} \mathscr{C})$. For $A \in \mathscr{L} \mathscr{C l}$ let $d(A)$ be the least positive interger $n$ for which $A \in \mathscr{C}_{n}$. Suppose $K$ is a ring having a large $\mathscr{T}$-ideal $I$ and $A$ is an ideal of $K$ with $d(A)=1$. Then $I \cap A$ is an $\mathscr{C}_{1}$-ideal of $I$, since $\mathscr{H}_{1}$ is hereditary, and hence an $\mathscr{L} \mathscr{C}$-ideal of $I$. Since $I \in \mathscr{T}, I \cap A=0$ and so $A=0$ by the largeness of $I$. Assume now that any ring containing a large $\mathscr{T}$-ideal contains no nonzero $\mathscr{C}_{i}$-ideals for $1 \leqq i \leqq k$. Let $K$ have a large $\mathscr{T}$ ideal $I$ and suppose $A$ is an ideal of $K$ with $d(A)=k+1$. Then $A \neq 0$ so that $J=I \cap A \neq 0$, and since $I \in \mathscr{G}, J$ is a $\mathscr{T}$-ideal of $A$. Select $N$ maximal relative to $N$ an ideal of $A$ and $N \cap J=0$. Then $J$ is a large $\mathscr{T}$-ideal of $A / N \neq 0$. But then $A / N$ can contain no nonzero $\mathscr{C}_{k}$-ideal and this contradicts $d(A)=k+1$. Thus $K$ has no $\mathscr{C l}_{k+1}$-ideals $\neq 0$ and the induction is complete. It follows that $\mathscr{L} \mathscr{M}$ is hereditary since $\mathscr{T}$ has property $(\lambda)$.

4. Subdirect sums. As shown by Kurosh [12], a class $\mathscr{S}$ of rings is a semisimple class if and only if it satisfies the two conditions:

(S1) If $K \in \mathscr{S}$ then every nonzero ideal of $K$ has a nonzero homomorphic image in $\mathscr{S}$.

(S2) If every nonzero ideal of a ring $K$ has a nonzero homomorphic image in $\mathscr{S}$ then $K \in \mathscr{S}$.

We provide another characterization in

Theorem 4.1. A class $\mathscr{S}$ of (associative) rings is a semi-simple class if and only if $\mathscr{S}$ satisfies the following:

(a) $\mathscr{S}$ is hereditary.

(b) $\mathscr{S}$ is closed under subdirect sums.

(c) $\mathscr{S}$ is closed under extensions: $K / A \in \mathscr{S}$ and $A \in \mathscr{S}$ implies $K \in \mathscr{S}$.

(d) If $I$ is an ideal of $K$ such that $0 \neq I / B \in \mathscr{S}$ for some ideal $B$ of $I$, then there is an ideal $A$ of $K$ with $A \subseteq I$ and $0 \neq I / A \in \mathscr{S}$.

Proof. Suppose that $\mathscr{S}$ is a semisimple class. That $\mathscr{S}$ is hereditary was shown in [4], while (b) was established in [12]. Also T. Jenkins has shown (c) in [11]. For (d), let $\mathscr{R}$ be the radical class for $\mathscr{S}$ and $I, K$ satisfy the conditions of (d). Then $I \notin \mathscr{R}$ so $r(I) \neq I$. Since $r(I)$ is an ideal of $K$ contained in $I$ we have $0 \neq I / r(I) \in \mathscr{S}$.

For the converse we note that by (a) $\mathscr{S}$ has property (S1). Let $K$ be a ring for which every nonzero ideal has a nonzero image in $\mathscr{S}$. 
Then $\left\{A_{\beta}: A_{\beta}\right.$ is an ideal of $K, 0 \neq K / A_{\beta} \in \mathscr{S}$ for $\left.\beta \in B\right\}$ is not empty. Let $M=\bigcap_{\beta \in B} A_{\beta}$. In $K / M, \bigcap_{\beta \in B}\left(A_{\beta} / M\right)=0$ so by (b) $K / M \in \mathscr{S}$, being a subdirect sum of the rings $K / A_{\beta} \cong(K / M) /\left(A_{\beta} / M\right)$ each of which is in $\mathscr{S}$. If $M \neq 0$, then $M$ has a nonzero image in $\mathscr{S}$ say $M / B^{\prime}$. By (d) $0 \neq M / A \in \mathscr{S}$ for $A$ an ideal of $K$. Then $K / M=(K / A) /(M / A)$ so that $K / A \in \mathscr{S}$ by (c). Hence $A=A_{\beta}$ for some $\beta \in B$ and so $M \cong A$. This contradiction establishes that $K \in \mathscr{S}$ so that $\mathscr{S}$ has property (S2).

Because of its connection to Theorem 4.1, we state without proof a result (unpublished) due to S. E. Dickson.

THEOREM 4.2. A class $\mathscr{S}$ of rings is a semisimple class for a radical class which contains all nilpotent rings if and only if $\mathscr{S}$ has properties (a), (b), (c) above, and in addition $\mathscr{P}$ contains no zero rings.

As is known not every radical class is hereditary. However, every radical class is closed under extensions as well as arbitrary direct sums. The majority of the well-known radical classes-e.g., Baer-McCoy, Levitzki, Nil, Jacobson, Brown-McCoy, (von Nuemann) Regular-are hereditary. Consequently, in view of [10], one might ask if these can serve as semisimple classes for radical classes. However, none of the above mentioned classes are closed under subdirect sums, as exemplified by the ring of integers in the case of the Regular rings, and the ring of even integers for the other classes [cf., Theorem 4.4 and Corollary 4.6 below]. Recall that a radical class is hypernilpotent if it is hereditary and contains all zero (hence all nilpotent) rings. We now show that no hypernilpotent radical class can be a semisimple class unless it consists of all rings.

Proposition 4.3. A hypernilpotent radical class $\mathscr{R}$ is a semisimple class if and only if it is closed under subdirect sums.

Proof. One way is clear, so assume $\mathscr{R}$ is closed under subdirect sums. We verify (d) of Theorem 4.1 to show that $\mathscr{R}$ is a semisimple class. Thus let $K$ have an ideal $I$ such that $0 \neq I / B \in \mathscr{R}$. If $B^{\prime}$ is the ideal of $K$ generated by $B$ then $B^{\prime} \cong I$. If $B^{\prime} \neq I, I / B^{\prime} \cong(I / B) /\left(B^{\prime} / B\right) \in \mathscr{R}$, while if $B^{\prime}=I$, then since $\left(B^{\prime}\right)^{3} \cong B, I^{2} \neq I$ so that $I / I^{2} \in \mathscr{R}$, being a zero ring.

THEOREM 4.4. If the hypernilpotent radical class $\mathscr{R}$ is a semisimple class, then $\mathscr{R}$ consists of all rings.

Proof. If $\mathscr{R}$ is a semisimple class it is closed under subdirect sums. Let $E$ denote the ring of even integers, $I$ the ring of all 
integers. Now if $A_{k}=\left(2^{k}\right)$ for $k \geqq 3$, then $E$ is a subdirect sum of the nilpotent rings $N_{k}=E / A_{k}$ so that $E \in \mathscr{R}$. Then since $E /(2 p) \cong$ $G F(p)$ for $p \geqq 3, p$ a prime, $G F(p) \in \mathscr{R}$ and hence $I \in \mathscr{R}$ being a subdirect sum of these. Now let $(K)_{n}$ denote the total $n \times n$ matrix ring over a ring $K$. Each $N_{k}$ is nilpotent so that $\left(N_{k}\right)_{n}$ is nilpotent and thus in $\mathscr{R}$. Then $\left(A_{k}\right)_{n}$ is an ideal of $(E)_{n}$ and $\left(N_{k}\right)_{n}=\left(E / A_{k}\right)_{n} \cong$ $(E)_{n} /\left(A_{k}\right)_{n}$, and this implies that $(E)_{n} \in \mathscr{R}$ for each $n \geqq 1$, since $\bigcap_{k \geqq 3}\left(A_{k}\right)_{n}=0$. It then follows that

$$
(E)_{n} /((2 p))_{n} \cong(E /(2 p))_{n} \cong(G F(p))_{n} \in \mathscr{R}
$$

for $p \geqq 3$ so that $(I)_{n} \in \mathscr{R}$ being a subdirect sum of the $(G F(p))_{n}$. Now Amitsur has shown [1, Th. 4] that every ring is a homomorphic image of a subdirect sum of total matrix rings of finite order over $I$, and this establishes the theorem.

Theorem 4.5. A hereditary radical class $\mathscr{R}$ is a semısimple class if and only if $\mathscr{R}$ is closed under subdirect sums.

Proof. Again we verify (d) of Theorem 4.1; further, we assume $\mathscr{R}$ is not hypernilpotent. Let $I$ be an ideal of a ring $K$ with $0 \neq I / B \in \mathscr{R}$. Letting $B^{\prime}$ ke the ideal generated by $B$ in $K$, we show that $B^{\prime} \neq B$ leads to a contradiction. Since $\left(B^{\prime} \mid B\right)^{3}=0, \mathscr{R}$ contains a zero ring $S \neq 0$. If any element of $S$ has infinite additive order, then $\mathscr{R}$ contains the zero ring $A_{0}$ on the infinite cyclic group and thus $\mathscr{R}$ contains all zero rings by [5], a contradiction. If $S$ has a torsion additive group then $\mathscr{R}$ contains a zero ring $A_{p}$ on the cyclic group of order $p$ for some prime $p$, and so $\mathscr{L} \mathscr{C} \subseteq \mathscr{R}$, where $\mathscr{C}=$ $\left\{0, A_{p}\right\}$. By [7, Lemma 1], $\mathscr{L} \mathscr{C l}=\mathscr{L} \mathscr{L} \cap \mathscr{C}_{p}$, where $\mathscr{G}_{p}$ consists of all rings having $p$-primary additive group. In $A_{0}, \bigcap_{n \geqq 1}\left(p^{n}\right)=0$ and $A_{0} /\left(p^{n}\right) \in \mathscr{L} \mathscr{Z} \cap \mathscr{S}_{p} \subseteq \mathscr{R}$ for each $n \geqq 1$. Thus again $A_{0} \in \mathscr{R}$, leading to a contradiction.

CoRollary 4.6. If $\mathscr{R}$ is a proper radical class which is also a semisimple class then $\mathscr{R} \subseteq \mathscr{S} \mathscr{Z} \cap \mathscr{U} \mathscr{Z}$. Thus $K \in \mathscr{R}$ and $I$ an ideal of $K$ implies $I^{2}=I$. Further $\mathscr{R}$ contains at most a finite number of distinct $G F(p)$ 's.

REMARK. We are unable at this time to obtain an example of a radical-semisimple class. One cannot simply take the class of all rings $K$ for which $I^{2}=I$ for all ideals $I$ of $K$, by the above corollary. On the other hand if one takes $\mathscr{C}=\{K, 0\}$, where $K$ is an idempotent simple ring with unit, then it is easy to show that $\prod_{i \geqq 1} K_{i}, K_{i}=K$, is not in $\mathscr{L} \mathscr{C}$. We conjecture that the class $\mathscr{R}=\mathscr{L} \mathscr{C}$, where $\mathscr{C l}$ consists of all idempotent simple rings without unit is a semi- 
simple class also; it is readily verified that $\mathscr{L} \mathscr{M}=\mathscr{M}_{2}$. In this connection the following may be useful:

Proposition 4.7. A hereditary radical class $\mathscr{R}$ is closed under subdirect sums if and only if in every ring $K$ there exists an ideal $I$ minimal relative to $K / I \in \mathscr{R}$. Moreover $I$ is unique.

Proof. Suppose $\mathscr{R}$ is closed under subdirect sums and for any $K$, let $M(K)=\bigcap I$, where the intersection is taken over all ideals $I$ of $K$ for which $K / I \in \mathscr{R}$ (note that $K$ is in this set). Then $M(K)$ is the required ideal since $R / M(K)$ is a subdirect sum of the rings $K / I$. Clearly it is unique.

Conversely, let $K$ be a subdirect sum of rings in $\mathscr{R}$. Then $K$ contains ideals $\left\{I_{\alpha}: \alpha \in A\right\}$ with $K / I_{\alpha} \in \mathscr{R}$ and $\bigcap_{\alpha \in A} I_{\alpha}=0$. Let $I$ be minimal with $K / I \in \mathscr{R}$. By the heredity of $\mathscr{R}$,

$$
I /\left(I \cap I_{\alpha}\right) \cong\left(I+I_{\alpha}\right) I_{\alpha} \in \mathscr{R}
$$

for each $\alpha \in A$. Using the extension property and an isomorphism theorem $K /\left(I \cap I_{\alpha}\right) \in \mathscr{R}$ for each $\alpha \in A$. Thus $I \cap I_{\alpha}=I$ for each $\alpha$ so $I \subseteq I_{\alpha}$ for each $\alpha \in A$. But then $I=0$ so that $K \in \mathscr{R}$.

\section{REFERENCES}

1. S. A. Amitsur, The identities of PI-rings, Proc. Amer. Math. Soc. 4 (1953), 27-34.

2. - Radicals in rings and bicategories, Amer. J. Math. 76 (1954), 100-125.

3. - A general theory of radicals, III. Applications, Amer. J. Math. 76 (1954), 126-136.

4. T. Anderson, N. Divinsky, and A. Sulinski, Hereditary radicals in associative and alternative rings, Canad. J. Math. 17 (1965), 594-603.

5. - Lower radical properties for associative and alternative rings, J. London Math. Soc. 41 (1966), 417-424.

6. E. P. Armendariz and W. G. Leavitt, Non-hereditary semisimple classes in Proc. Amer. Math. Soc. 18 (1967), 1114-1117.

7. - The hereditary property in the lower radical construction, Canad. J. Math. 20 (1968), 474-476.

8. S. E. Dickson, A torsion theory for Abelian categories, Trans. Amer. Math. Soc. 121 (1966), 223-235.

9. A. E. Hoffman and W. G. Leavitt, Properties inherited by the lower radical (to appear)

10. J. P. Jans, Some aspects of torsion, Pacific J. Math. 15 (1965), 1249-1259.

11. T. L. Jenkins, The theory of radicals and radical rings, $\mathrm{Ph}$. D. Thesis, Univ. of Nebraska, 1966.

12. A. G. Kurosh, Radicals of rings and algebras, Mat. Sbornik (75) 33 (1953), 13-26 (Russian).

Received December 20, 1966.

UNIVERSITY OF TEXAS

Austin, Texas 



\section{PACIFIC JOURNAL OF MATHEMATICS}

\section{EDITORS}

\author{
H. ROYDEN \\ Stanford University \\ Stanford, California \\ R. R. Phelps \\ University of Washington \\ Seattle, Washington 98105
}

J. DUGUNDJI

Department of Mathematics

University of Southern California

Los Angeles, California 90007

RICHARD ARENS

University of California

Los Angeles, California 90024

\section{ASSOCIATE EDITORS}
E. F. BECKENBACH
B. H. NeumanN
F. WOLF
K. YosidA

\section{SUPPORTING INSTITUTIONS}

UNIVERSITY OF BRITISH COLUMBIA

CALIFORNIA INSTITUTE OF TECHNOLOGY

UNIVERSITY OF CALIFORNIA

MONTANA STATE UNIVERSITY

UNIVERSITY OF NEVADA

NEW MEXICO STATE UNIVERSITY

OREGON STATE UNIVERSITY

UNIVERSITY OF OREGON

OSAKA UNIVERSITY

UNIVERSITY OF SOUTHERN CALIFORNIA
STANFORD UNIVERSITY

UNIVERSITY OF TOKYO

UNIVERSITY OF UTAH

WASHINGTON STATE UNIVERSITY

UNIVERSITY OF WASHINGTON

AMERICAN MATHEMATICAL SOCIETY CHEVRON RESEARCH CORPORATION TRW SYSTEMS

NAVAL WEAPONS CENTER

Printed in Japan by International Academic Printing Co., Ltd., Tokyo, Japan 


\section{Pacific Journal of Mathematics}

\section{Vol. 26, No. $1 \quad$ November, 1968}

Efraim Pacillas Armendariz, Closure properties in radical theory......... 1

Friedrich-Wilhelm Bauer, Postnikov-decompositions of functors .......... 9

Thomas $\mathrm{Ru}-$ Wen Chow, The equivalence of group invariant positive definite

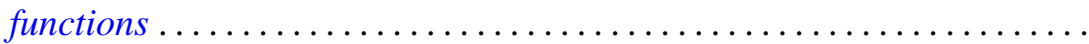

Thomas Allan Cootz, A maximum principle and geometric properties of

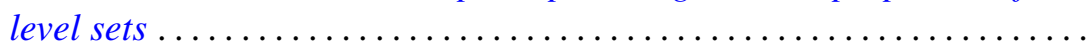

Rodolfo DeSapio, Almost diffeomorphisms of manifolds ............ 47

R. L. Duncan, Some continuity properties of the Schnirelmann density......

Ralph Jasper Faudree, Jr., Automorphism groups of finite subgroups of

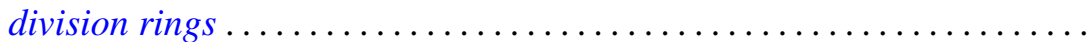

Thomas Alastair Gillespie, An invariant subspace theorem of $J$.

Feldman.........................................

George Isaac Glauberman and John Griggs Thompson, Weakly closed direct factors of Sylow subgroups .............................

Hiroshi Haruki, On inequalities generalizing a Pythagorean functional equation and Jensen's functional equation .....................

David Wilson Henderson, D-dimension. I. A new transfinite dimension.....

David Wilson Henderson, D-dimension. II. Separable spaces and

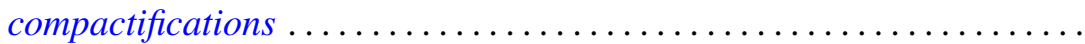

Julien O. Hennefeld, A note on the Arens products ............... 115

Richard Vincent Kadison, Strong continuity of operator functions ...

J. G. Kalbfleisch and Ralph Gordon Stanton, Maximal and minimal coverings of $(k-1)$-tuples by $k$-tuples.

Franklin Lowenthal, On generating subgroups of the Moebius group by pairs of infinitesimal transformations...

Michael Barry Marcus, Gaussian processes with stationary increments possessing discontinuous sample paths . .

Zalman Rubinstein, On a problem of Ilyeff ...

Bernard Russo, Unimodular contractions in Hilbert space. ...

David Lee Skoug, Generalized Ilstow and Feynman integrals...

William Charles Waterhouse, Dual groups of vector spaces . 